\title{
A New Form of Damage to PMMA Intraocular Lenses by Nd:YAG Laser Photodisruptors
}

\author{
J. MELLERIO, ${ }^{1}$ M. R. C. CAPON,${ }^{2}$ F. DOCCHIO,${ }^{3}$ D. SLINEY ${ }^{4}$ and J. KRAFFT ${ }^{2}$ \\ London; Brescia, Italy; and Maryland, USA
}

\begin{abstract}
Summary
The damage that Nd:YAG laser radiation can cause to plastic (PMMA) IOLs during pseudophakic capsulotomy is well documented in the literature. This damage is the result of direct plasma action on the lens material. We report here another form of damage to PMMA IOLs which is more subtle and does not result from plasma action in the plastic. Even when the irradiance within the IOL is well below the threshold for optical breakdown, the PMMA can be damaged if irradiated by many laser pulses. This subthreshold damage is therefore cumulative. Its importance, likelihood of occurrence and ways to minimise this are considered.
\end{abstract}

The Nd:YAG photodisruptor is now regarded as an important ophthalmic tool and is in widespread use. The complications and hazards of its use in treatment regimes have been extensively reported. ${ }^{1}$ Since the laser was first introduced damage from direct impact of its plasma on or within intraocular lenses (IOLs) has been known and the implications considered.1,2 Damage from direct plasma impact (optical breakdown) is most likely to occur during capsulotomy or iridotomy for one of three reasons. First, inaccurate focusing can place the breakdown in unwanted sites; secondly, if the aiming beam and the laser beam are not confocal aiming will not be correct. Thirdly, there is the problem of 'plasma wander' where the plasma may occur anywhere within a sizeable volume of the beam within the beam waist at the focus (Fig. 1). This latter situation is largely beyond the control of even the most competent surgeon. It is most likely to occur when the laser energy is increased to disrupt a thickened region within a posterior capsule. The hazardous volume shown in Figure 1 becomes extended and a second plasma may form on the rear face of the IOL as well as at the laser focus: this possibility is increased because interfaces lower the thresholds for optical breakdown. ${ }^{3,4}$

A different type of damage has been reported to occur in polymethylmethacrylate (PMMA), from which most intraocular lenses are made. PMMA has been widely used by physicists for the fabrication of optical components for many years and with the introduction of lasers these components were found to be easily damaged by radiant fluxes that were high but below that at which optical breakdown occurs. This form of

From ${ }^{1}$ School of Paramedical Studies, Polytechnic of Central London, 115 New Cavendish Street, London, W1M 8JS, UK

2Department of Clinical Ophthalmology, Institute of Ophthalmology, Judd Street, London, WC1H 9QS, UK ${ }^{3}$ Dipartimento di Automazione Industriale, Facolta di Ingegneria Meccanica, Universita di Brescia, 25060 Brescia, Italy

${ }^{4}$ Laser Microwave Division, US Army Environmental Hygiene Agency, Aberdeen Proving Ground, Maryland, USA

Correspondence: J. Mellerio, School of Paramedical Studies, Polytechnic of Central London, 115 Cavendish Street, London W1M 8JS

Presented at the Annual Congress of the Ophthalmological Society of the United Kingdom, April 1988 
damage has been well described in the Russian literature ${ }^{5,6}$ and ways of reducing it have been studied. ${ }^{7}$ A question of ophthalmic importance follows from this knowledge: if PMMA can be damaged without plasma formation in the physics laboratory could it be damaged by Nd:YAG laser radiation at levels used in the clinic?

We have investigated the action of Nd:YAG laser energy on PMMA IOLs to determine if cumulative damage could be produced by ophthalmic lasers at points in the beam remote from the region in which plasmas were formed. We describe our experiments with three types of laser found in the clinic and report that this damage can occur to IOLs in situations which are of clinical interest. We offer an estimate of the conditions that are likely to be hazardous and suggest ways in which these might be

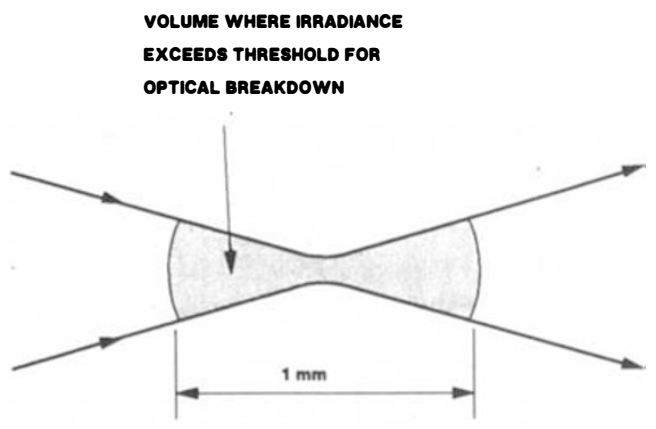

MatituTe of OPHTMALMOLOQV, LONOON 1000

Fig. 1. Diagram shows volume around beam focus at which the irradiance (energy/unit area/unit time) is sufficient for a plasma to occur. This volume can be up to $1 \mathrm{~mm}$ in length. From shot-to-shot plasmas will form at different sites within this volume: this is called plasma wander.
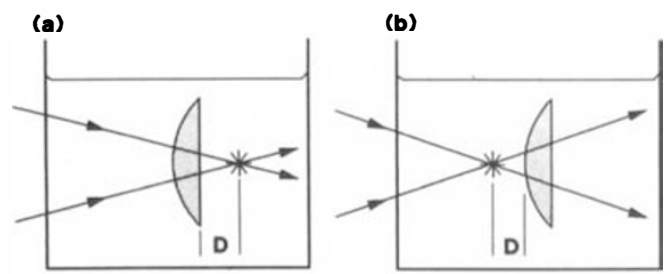

Fig. 2. Diagram to show the experimental setups. The PMMA intraocular lens was positioned in a water bath and the laser energy was focused (a) downstream, or (b) upstream, a distance $D$ from the nearest surface of the IOL. mitigated. This paper follows two preliminary reports of this form of damage ${ }^{8,9}$ and a more detailed investigation comparing this form of damage with that from direct impact in bulk PMMA. ${ }^{10}$

\section{Methods}

Three commercially available Nd:YAG photodisruptors were used. The first was a large water-cooled, actively Q-switched instrument with a pulse length of $15 \mathrm{~ns}$ (FWHM) operating in the fundamental transverse mode with a spot diameter of 30 microns in air. The second was a small aircooled, passively Q-switched device with a pulse length of $12 \mathrm{~ns}$ (FWHM) operating in a low order mode with a spot diameter of 30 microns in air. The third laser was modelocked with a spot size in air of 25 microns. The energy meters on each laser were checked against either an EG\&G series 581 radiometer or a Gen Tec ED-200 joulemeter and oscilloscope. The threshold energy for $100 \%$ probability of plasma formation in distilled water was determined as described elsewhere. ${ }^{10}$ For exposing the lenses, an output energy of about 1.5 times this threshold was used.

Reject PMMA IOLs were viewed under a high power dissecting microscope and the presence of any visible flaws was noted. Each IOL was then positioned in distilled water orthogonal to the laser beam axis which was focused either upstream from the lens front surface or downstream from the lens posterior surface (Fig. 2). It must be stressed that with this configuration a plasma was never formed in, on or adjacent to the lens and this was checked by conducting the exposures in a dimly lit room and with a dark adapted observer looking at the plasmas. In each case, the entire laser beam always fell completely within the IOL. For each value of $\mathrm{D}$, the plasma to lens surface distance (Fig. 2), a preset number of $\mathrm{Nd}: \mathrm{YAG}$ pulses were fired and the energy output of the pulses was monitored.

After irradiation, the IOL was macrophotographed in white light, sectioned through the irradiated volume and gold coated prior to examination by scanning electron microscopy (SEM). 


\section{Results}

For the two Q-switched lasers the irradiance of the beam cross section occupied by the IOL was calculated for each distance D (Fig. 2). It was always at least an order of magnitude below the threshold for optical breakdown in PMMA ${ }^{10,11}$ as is shown in Table I. This Table also shows that cumulative subthreshold damage was caused when the IOL was 1 and $4 \mathrm{~mm}$ but not at 10 $\mathrm{mm}$ upstream from the plasma. Damage also occurred when the plasma was $1 \mathrm{~mm}$ in front of the IOL. Damage was produced by 100 shots when the plasma was $1 \mathrm{~mm}$ either upstream or downstream, but not with 25 shots. At $4 \mathrm{~mm} 200$ shots caused damage but even 1000 shots at $10 \mathrm{~mm}$ did not.

With the mode-locked laser cumulative damage was found when the lens was $4 \mathrm{~mm}$ upstream from the plasma. With this laser the experimental protocol was slightly different to that for the Q-switched instruments: several series of 1000 shots were given, each series at a different energy value, with the IOL always $4 \mathrm{~mm}$ upstream from the plasma. Even with an energy per pulse of 1.2 $\mathrm{mJ}$, i.e. about 1.5 times the threshold for optical breakdown in PMMA, damage was seen within the IOL. If the Q-switched or mode-locked laser beam was moved after each shot so that it passed through a fresh part of the IOL damage was not found.

The damage was readily visible by macrophotography as blemishes within the plastic (Fig. 3), was always within the irradiated volume of the IOL and was at its

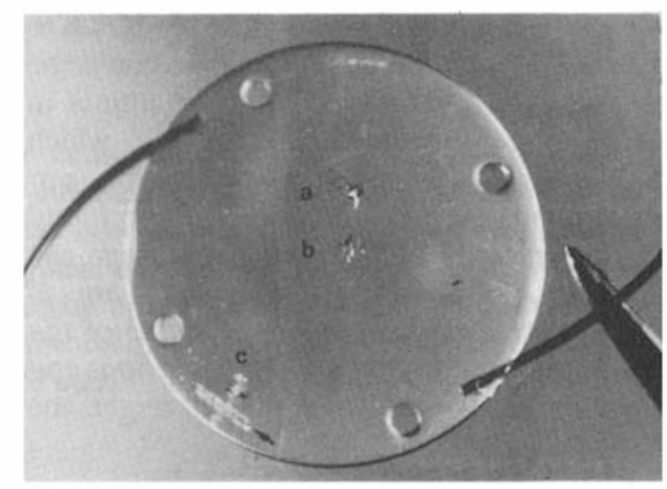

Fig. 3. Macrophotograph of a PMMA IOL damaged by a mode-locked Nd:YAG laser using the experimental setup in Fig. $2 a$ with $D=+4 \mathrm{~mm}$. Three areas of damage are shown ( $a, b$ and $c)$ for three different series of shots with the IOL moved within the beam between each series. Scratch mark indicated lens polarity (arrow).

Table. I Occurrence of damage to PMMA intraocular lenses by Q-switched Nd:YAG laser photodisruptors. Symbol (+) indicates the laser focus was downstream and (-) the focus upstream, from the IOL (Fig. 2). W indicates the laser was water-cooled and $A$ that it was air-cooled. The calculated irradiance for each shot to the IOLs was below the threshold for optical breakdown, $10^{10} \mathrm{~W} / \mathrm{cm}^{2} .{ }^{10} \mathrm{For}(-)$ values of $D$ the calculated irradiances assume $100 \%$ of the incident energy was transmitted beyond the plasma to the IOL.

\begin{tabular}{cccccc}
\hline $\begin{array}{c}\text { Distance }(D) \\
\text { to focus from } \\
\text { IOL }(\mathrm{mm})\end{array}$ & $\begin{array}{c}\text { Number } \\
\text { of } \\
\text { shots }\end{array}$ & $\begin{array}{c}\text { Average } \\
\text { energy per } \\
\text { shot }(\mathrm{mJ})\end{array}$ & $\begin{array}{c}\text { Laser } \\
\text { type }\end{array}$ & $\begin{array}{c}\text { Calculated } \\
\text { irradiance } \\
\text { at IOL } \\
\left.\left(\times 10^{8} \text { W/cm }\right)^{2}\right)\end{array}$ & $\begin{array}{c}\text { Damage } \\
(\mathrm{Y} / \mathrm{N})\end{array}$ \\
\hline+1 & 25 & 6 & $\mathrm{~A}$ & 8 & $\mathrm{~N}$ \\
+1 & 100 & 7.5 & $\mathrm{~W}$ & 8 & $\mathrm{Y}$ \\
+4 & 1000 & 6 & $\mathrm{~A}$ & 4 & $\mathrm{Y}$ \\
+4 & 578 & 7 & $\mathrm{~W}$ & 3 & $\mathrm{Y}$ \\
+4 & 200 & 7.5 & $\mathrm{~W}$ & 3 & $\mathrm{Y}$ \\
+10 & 1000 & 6 & $\mathrm{~A}$ & 8 & $\mathrm{~N}$ \\
-1 & 100 & 7 & $\mathrm{~A}$ & 9 & $\mathrm{Y}$ \\
-1 & 100 & 7.5 & $\mathrm{~W}$ & 8 & $\mathrm{Y}$ \\
-1 & 25 & 8 & $\mathrm{~W}$ & 9 & $\mathrm{~N}$ \\
\hline
\end{tabular}


(a)

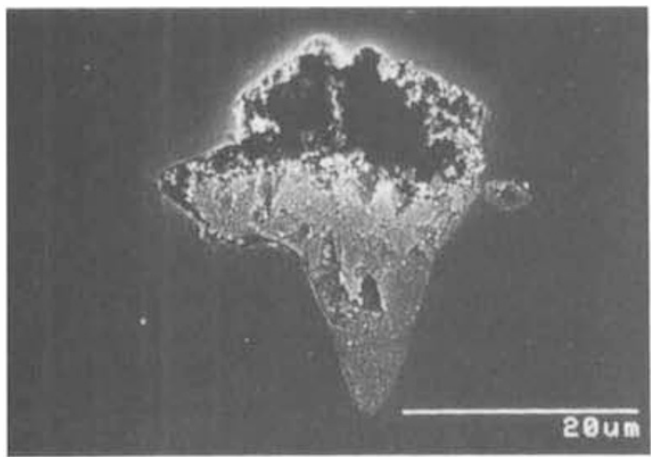

(b)

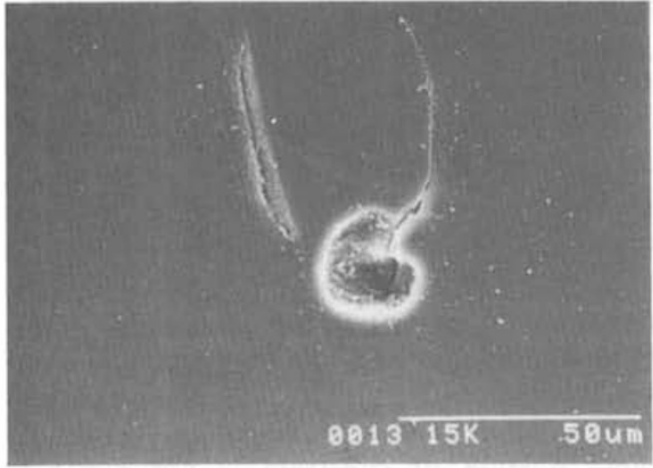

(c)

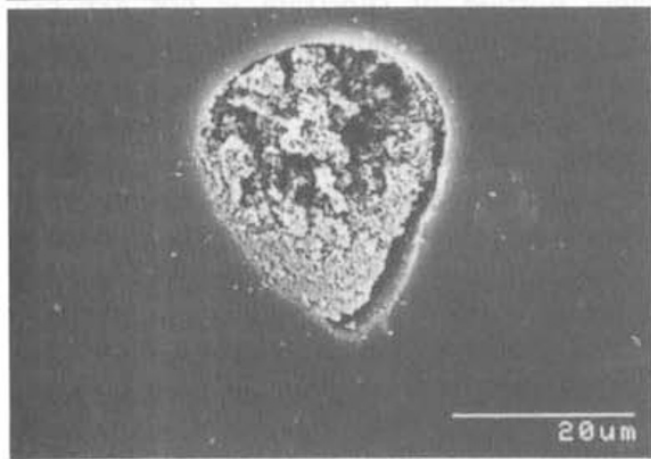

(d)

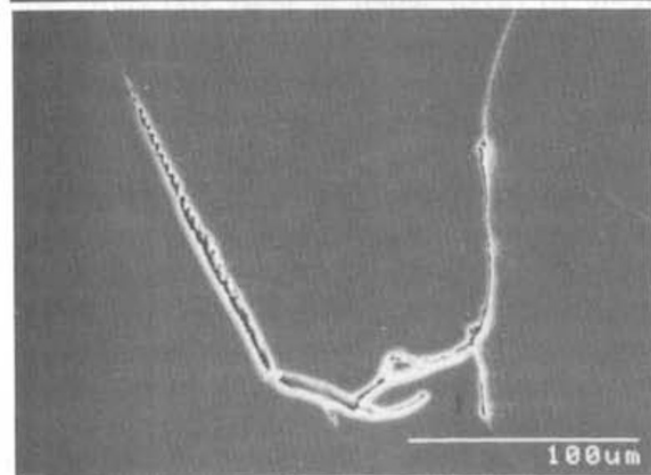

Fig. 4. (a,b) SEM of IOL damaged by $Q$-switched $N d: Y A G$ laser; (c,d) SEM of IOL damaged by mode-locked $N d: Y A G$ laser. maximum in what had been the central portion of the laser beam. With SEM the form of the damage induced by all three laser types (Q-switched and mode-locked) was seen to be similar and is shown in Figure 4. Both laser types produced cavities and cracks within the IOL: most of the cavities contained debris. These cavities were small and were formed first; cracks spread out from the cavities as more laser shots were fired and they could become quite extensive with high shot numbers (Fig. 4d). In no case was damage produced at the surface of a lens and the damage was of a different form to that produced by a plasma deep within, on or near to an IOL. ${ }^{12}$

\section{Discussion}

The processes that lead to the formation of a plasma in PMMA have been discussed previously $y^{4,13}$ and can only be understood if a probabilistic view point is adopted. The damage associated with plasmas will therefore also occur in a statistical way. ${ }^{14}$ The type of subthreshold damage that we report here, although it is not due to plasma formation, also demonstrates probabilistic features. The irradiance in the plastic is below the threshold for optical breakdown but micro-impurities, which will always be present, absorb some of the laser energy and are thus heated and transformed into larger, more absorptive inclusions. ${ }^{5-7}$ This heating may lead to greater absorption from the next laser pulse which leads in turn to further heating, enlargement of the inclusion, and so on. Eventually, with further input of laser energy, a nucleus of changed substrate is formed which is of sufficient size to qualify as visible damage. The incidence of damage, and its distribution, will therefore follow that of the micro-impurities and their absorption characteristics; and both these will be dispersed in a random way. Consequently, it is to be expected that the quality of the plastic substrate will influence the occurrence rates of cumulative subthreshold damage. ${ }^{10}$ That local heating is a cause of the damage is borne out by the damage seen with SEM. The cavities contain granular debris which may fall out in the sectioning process and there is no evidence of the melting and 
explosive disruption that are obvious if the site of a plasma is examined.

It is interesting to note that the criteria for damage formation in Table $I$ are approximately the same for a given distance whether the plasma is upstream or down stream from the IOL surface. This is yet another demonstration that the shielding effect of a plasma is small at clinically used energies. This phenomenon is discussed further by Capon et al. ${ }^{15}$

It may be thought that in a pseudophakic eye provided the laser focus is well away from the IOL, there is no risk of lens damage. Such a situation might typically be when disruption of a vitreous membrane is attempted. If the focal plane was $4 \mathrm{~mm}$ behind the lens, with a typical photodisruptor the laser beam would be about $1 \mathrm{~mm}$ in diameter at the lens so the irradiance would be well below that required for plasma formation. However, a large number of shots might be used and the possibility of cumulative damage arises. This will be especially true if the laser beam passes through the same volume of the IOL for each shot. There is no evidence that restorative processes operate in PMMA so summation of the damage from a number of treatment sessions would extend throughout the life time of the implant.

The form of cumulative damage in an IOL suggests that there would be no risk of the lens disrupting or splintering. As the damage is deep in the lens, there would be no risk either of monomer or toxin release, if this is ever a risk with PMMA. ${ }^{2}$ The cracks and cavities that are typical of this damage would act as scattering entities and as they are most likely to be distributed in the centre of the IOL, their effect would be greatest in bright light when the pupil is small. The magnitude of visual disability due to this glare would probably not be great but the possibility of its occurrence should be considered. A more insidious problem is that the cracks and cavities will lower the threshold for optical breakdown and plasma formation from subsequent photodisruptor treatment even if the beam focus is several millimetres from the IOL.

The demonstration of subthreshold cumulative damage in PMMA IOLs raises a number of questions. The first is, does this kind of damage occur in IOLs made from substrates other than PMMA? In theory, it should be expected but the resistance of a . material to the damaging processes probably varies. The extent of this variation is currently under investigation.

The second question is, does this type of damage form in the crystalline lens or the cornea? We have repeated, with a Qswitched laser, the experiments described here on porcine lenses. We found no damage when the plasma was $4 \mathrm{~mm}$ down stream from the posterior surface of the lens even with 1000 shots at 4 times the threshold for optical breakdown in the lens substance. Similar findings were made with porcine corneas. It must be concluded that biological tissues are more resistant to subthreshold cumulative damage probably because they have a lower dielectric strength than plastics and partly because they are more flexible and fluid so that, at the submicroscopic level, the same portion of substrate is not exposed repeatedly to the many shots.

The third question is whether a clinically useful threshold can be defined and appropriate guidelines proposed? For Qswitched lasers (the most popular), interpolation from Table I would suggest that an approximate safe upper limit to the number of shots is related to the IOL to plasma distance. This limit will also depend upon laser energy per pulse. Table I shows, approximately, that this damage is most likely to occur when at least 100 shots are fired at a target in the anterior or midvitreous cavity. Currently, we are working to refine this Table for IOLs made from different materials. The cumulative nature of this damage has been clearly demonstrated so a set of guidelines can be proposed: they are briefly outlined below and would apply to any procedure where the $\mathrm{Nd}: \mathrm{YAG}$. beam passes through the IOL but is focused remote from the lens either upstream or downstream.

(a) Before treatment inspect the IOL carefully for blemishes (these might have been formed by earlier Nd:YAG 
treatment) and if possible ensure that the laser beam passes through an unmarked portion of the lens;

(b) Use the minimum laser energy per pulse which achieves the desired effect and as few shots as possible;

(c) To avoid summation, try to move the laser beam every few shots so that it passes through a part of the IOL that was not previously exposed.

Moving the Nd:YAG laser beam to fresh parts of the IOL every few shots may be difficult to achieve especially when working in the posterior vitreous cavity. In this case the beam may be up to 2 millimetres diameter at the IOL and exposures through virgin plastic may be prevented by vignetting at the pupil margin.

Our thanks are due to the Wellcome Foundation who provided financial support for MC and to the laser manufacturers who kindly allowed us to use their lasers.

\section{References}

${ }^{1}$ Steinert RF and Puliafito CA: The $N d: Y A G$ laser in Ophthalmology Philadelphia. W. B. Saunders, 1985.

2 Terry AC, Stark WJ, Newsome DA, Maumenee AE, Pina AE: Tissue toxicity of laser-damaged IOL implants. Ophthalmol 1985, 92: 414-69.

${ }^{3}$ Vogel A, Hentschel W, Holzfuss J, Lauterborn W: Cavitation bubble dynamics and acoustic transient generation in ocular surgery with pulsed Nd:YAG lasers. Ophthalmol 1986, 93: 1259-69.

${ }^{4}$ Bloembergen N. Laser-induced electric breakdown in solids. IEEE J Quant Elect 1974, QE10: $375-86$.

${ }^{5}$ Manenkov AA, Matyushin GA, Nechitalo VS,
Prokhoro AM, Tsaprilov AS: Nature of the cumulative effect in laser damage to optical materials. Sov J Quant Elect 1983, 13: 1580-3.

${ }^{6}$ Dyumaev KM, Manenkov AA, Maslyukov AP, Matyushin GA, Nechiailo VS, Prokhorov AM: Transparent polymers: a new class of optical materials for lasers. Sov J Quant Elect 1983, 13: 503-7.

${ }^{7}$ O'Connell RM, Romberger AB, Shaffer AA, Saito TT, Deaton TF, Seigenthaler KE: Improved laser-damage-resistant polymethylmethacrylate. J Opt Soc Am B 1984, 1: 853856.

${ }^{8}$ Capon M, Mellerio J, Docchio F: Damage to IOLs by Nd:YAG laser pulses focused in the vitreous. I. Q-switched lasers. J Cataract Refract Surg 1988 (In Press).

${ }^{9}$ Sliney DH, Dolch BR, Rosen A, Dejacma FW: Damage to IOLs by Nd:YAG lasers focused in the vitreous. II. Mode-locked lasers. $J$ Cataract Refract Surg 1988 (In Press).

${ }^{10}$ Doccio F, Mellerio J, Sliney D, Regondi P, Capon M: A study of laser-induced damage mechanisms in plastics used for intraocular lenses. Lasers in Ophthalmology 1988. (In Press).

${ }^{11}$ Bath PE, Romberger AB, Brown P: A comparison of Nd:YAG laser damage thresholds for PMMA and silicone intraocular lenses. Invest Ophthalmol Vis Sci 1986, 27: 795-8.

12 Davi SK, Gaasterland DE, Cummins CE, Liesegang G: Pulsed laser damage thresholds in vitro for intraocular lenses and membranes. IEEE J Quant Elect 1984, QE-20: 1458-64.

${ }^{13}$ Bass M, and Barrett $\mathrm{HH}$ : Avalanche breakdown and the probabilistic nature of laser-induced damage. IEEE J Quant Elect 1972, QE-8: 338-43.

${ }^{14}$ Manenkov AA, Matysuhin GA, Nechitailo VS, Prokhorov AM, Tsaprilov AS: Statistics of laser damage to transparent polymers. Sov $J$ Quant Elect 1983, 13: 884-6.

15 Capon M, Docchio F, Mellerio J: Graefes Arch Clin Exp Ophthalmol 1988 (in press). 